ISSN (O) 2589-8779 | (P) 2589-8760

CMRO 03 (10), 692-701 (2020)

\title{
An Argument for the Use of Amantadine to Treat Complex Regional Pain Syndrome
}

\author{
Robert G. Smith ${ }^{*}$
}

${ }^{1} \mathrm{DPM}, \mathrm{MSc}, \mathrm{RPh}$

\section{1 | INTRODUCTION}

A published definition for complex regional pain syndrome (CRPS), which states that CRPS is a form of spontaneous or stimulusinduced chronic pain that affects one limb in either the upper or lower extremity. This usually occurs after an injury and lasts for over six months (1, 2). CRPS occurs in patients who have undergone limb surgery, limb fractures, or trauma (1) and is characterised by severe, prolonged pain, changes in skin colour and temperature, and swelling and bone loss in the affected limb (1-3). CRPS is divided into two types: I and II. Patients who have reflex sympathetic dystrophy syndrome without confirmed nerve injury are categorised as having CRPS-I (1-4). On the other hand, CRPS-II, also known as causalgia, occurs when there is associated and established nerve damage (1-4). Eldufani et al. conducted a review of updated therapeutic strategies based on CRPS pathophysiology, presenting novel approaches and techniques for managing the disease (1).

Acerra et al. asserted that, despite being different conditions, phantom limb pain (PLP), stroke, and CRPS-I share some important similarities (5). Furthermore, based on these findings, they presented established principles of stroke rehabilitation and inferred that these principles are applicable to the rehabilitation of CRPS-I and PLP (5). Last, they proposed that it may be possible to adapt and apply treatments across all three disease states (i.e. post-stroke, CRPS-I, and PLP) because the patients share common neuroimaging, clinical findings, and the best recovery appears to be associated with a supreme return to normal brain function (5) There are few published studies on amantadine, dextromethorphan, and memantine in CRPS patients. The concept of (N-Methyl-D-aspartate) NMDA antagonism as a therapeutic target in neuropathic pain remains sound (6). There is a strong need for more studies on CRPS as well as the development of newer agents with fewer side effects on the central nervous system (6). The central theme of this review is to introduce the hypothesis that amantadine may be used to treat complex regional pain syndrome. First, the details of a literature search focused on amantadine and memantine as treatments for CRPS are presented. Second, the pharmacology of amantadine as it applies to inflammation, pain syndromes, and the pathology of CRPS is presented. Last, a comparison between amantadine and memantine and other

Supplementary information The online version of this article (https://doi.org/10.15520/jcmro.v3i10.35 3) contains supplementary material, which is available to authorized users.

Corresponding Author: Robert G. Smith $D P M, M S c, R P h$

Email:asamaan@cfl.rr.com 
NMDA receptor antagonists as treatments for CRPS is presented.

\section{2 | DATABASE SEARCH}

A search profile was compiled using key terms, and Boolean logic electronic searches were performed to identify relevant primary literature citations from 1967 to 2020 Figure 1. Further, a manual review of the citations' reference lists and bibliographies was undertaken to gather any additional information that could lead to further material for this review. Citations $(n=531)$ were reviewed and evaluated as defined by the search limitations. Accounting for duplication, $73.8 \%(n=392)$ of the citations were reviewed for significance and relevance before their inclusion in this review. The databases searched were PubMed, MEDLINE, and Cochrane, and the following keywords were used: "CRPS", "pain", "inflammation" "future treatment", "lower extremity", "amantadine", and "NMDA antagonists". The literature selected was focused on CRPS in patients who suffered from severe limb pain after surgery or trauma. Reports on other kinds of pain were excluded. The search was related to published studies over the last two decades and identified those that assessed the updated management and pathophysiology of CRPS. The literature was reviewed and evaluated for quality and relevance to the use of amantadine for CRPS of the lower extremity.

\section{3 | HISTORICAL ASPECTS OF CRPS}

In 1864, Silas Weir Mitchell reported of patients whose disease corresponded to what we now call CRPS-II (Causalgia) (7). In 1901, Paul Sudeck described this as "acute reflex bone atrophy after inflammation and injuries of the extremities and their clinical appearances", which corresponds to CRPS-I without nerve lesion (8). The next milestone in CRPS history was reached in 1936 by James A. Evans who coined the phrase "reflex sympathetic dystrophy", which was then used for several decades (9). Then, at a 1995 conference in Orlando, the use of the descriptive phrase "complex regional pain syndrome" was agreed upon to avoid claims about pathophysiology (10).

Apart from its various names, complex regional pain syndrome (CRPS) has also had different criteria for diagnosis over the years. The older criteria were proposed by Kozin et al. in 1981, Veldman et al. in 1993, and van de Beek et al. in 2002, none of which were subjected to rigorous testing of their psychometric properties (11-13). In an effort to be more definitive and consistent in the diagnosis of CRPS, the International Association for the Study of Pain (IASP) criteria and the Budapest criteria were proposed $(3,10,14)$. While the IASP criteria have high sensitivity but low specificity, the Budapest criteria appear to have better characteristics $(3,10-15)$. A validation study noted that the IASP criteria's high diagnostic sensitivity but low specificity result in a relatively high rate of false positive diagnoses and unnecessary treatments (15). The Budapest criteria, on the other hand, show the same high sensitivity but with improved specificity; therefore, they are recommended in both clinical and research settings (16). There are two types of Budapest criteria: clinical and research diagnostic. Overall, reflecting on the historical narrative described above, it took approximately 100 years for the acronym "CRPS" to be established.

\section{I CRPS AND INFLAMMATION}

Eldufani et al. asserted that there is universal agreement that CRPS is caused by a multifactorial process involving both the peripheral and central nervous systems $(1,17)$. For the purpose of this review, the emphasis on inflammation will be accentuated. CRPS patients exhibit all the signs of inflammation such as heat, pain, redness, and swelling (18). Several CRPS clinical trials have demonstrated symptoms that have been significantly improved by corticosteroids, suggesting that inflammatory mechanisms contribute to CRPS, especially in the acute phase (18-20). The presence of Langerhans cells in skin biopsies from CRPS patients as well as observed cellular infiltration, mainly of the lymphocytes, in synovial biopsy specimens have been reported (1721). Also, the inflammatory process that contributes 
AN ARGUMENT FOR THE USE OF AMANTADINE TO TREAT COMPLEX REGIONAL PAIN SYNDROME

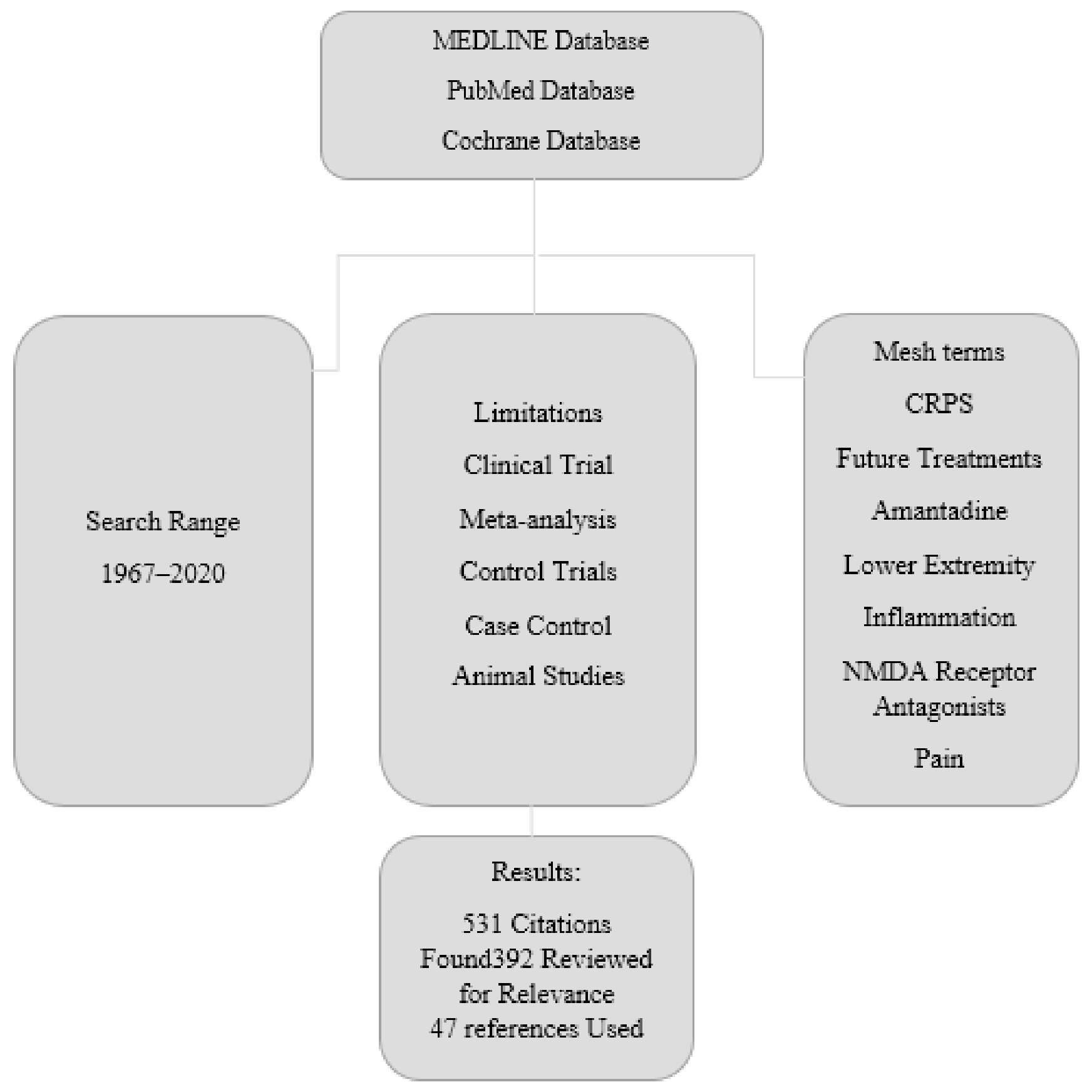

FIGURE 1: Citations ( $n=531$ ) were reviewed and evaluatedas defined by the search limitations. Accounting for duplication, $73.8 \%(n=392)$ of the citations were reviewed for significance and relevance for inclusion inthis review. 
to CRPS might result from two inflammatory cascades (21-24). The first is the classic inflammatory mechanism through the actions of immune cells such as mast cells and lymphocytes (22). Post-traumatic soft tissues, mast cells and lymphocytes release proinflammatory cytokines, including tumour necrosis factor alpha (TNF- $\alpha)$, interleukin- $1 \beta, 2$, and 6. Subsequently, these substances elevate plasma extravasation and cause localised edema (22). The second is the neurogenic inflammation mechanism through the direct release of neuropeptides and proinflammatory cytokines from nociceptive fibres, contributing to CRPS in response to different triggers, e.g. nerve injury (23). Neuropeptide mediators (calcitonin gene-related peptide (CGRP), substance $\mathrm{P}$, and bradykinin) increase plasma extravasation and cause vasodilation causing warm, red, and edema (24). In addition, the concept of neurogenic inflammation includes distinct classes of C-fibres with an afferent mediation function of pain and itch and an efferent neuro-secretory function (24).

Moreover, König S. et al. demonstrated that although biomarkers are lacking in CRPS, several inflammatory substances have been associated with the pathophysiology (24). Last, Kingery investigated neuropeptide, cytokine, and growth factor signaling in CRPS The objective of the study was to identify how neurocutaneous signaling is facilitated after fracture and examine how this altered signaling contributes to the development of CRPS-like changes in the injured limb (25). In a rat fracture model, the substance P (SP) evoked extravasation and edema responses that were enhanced in the fractured hind paw skin, leading to the postulate that neuokinin-1 (NK1) receptor upregulation mediates the facilitated extravasation and edema responses observed after SP injection. The NK1 receptor antagonist reversed hind paw warmth and edema and increased the vascular permeability, allodynia, and unweighting observed after tibia fracture in rats (25). There was also increased keratinocyte proliferation and NK1 receptor expression in the fractured hind paw (25). Similar to the rat fracture model, increased epidermal thickness and keratinocyte NK1 expression were observed in skin biopsies from CRPS patients (26). There was an up-regulation of inflammatory cytokine expression in the rat hind paw skin and in the keratinocytes at
4 weeks post-fracture (25). It was concluded that these inflammatory mediators appear to play a crucial role in the development of pain behaviour after fracture (26).

CRPS is a visible pain disease. There has been significant progress in understanding the pathophysiology, which will lead to better, individualised treatment with the inclusion of a variety of pharmacological agents such as pain relievers, antidepressants and anticonvulsants, corticosteroids, boneloss medications, sympathetic nerve-blocking medication, and intravenous ketamine, an N-methyl-Daspartate (NMDA) antagonism (6).

\section{5 | AMANTADINE AND PAIN TREATMENTS}

The N-methyl-D-aspartate (NMDA) receptor is a ligand-gated channel whose major endogenous agonist is glutamate, which is the predominant excitatory neurotransmitter in the Central Nervous System (CNS). When this receptor is inhibited, a decrease in the neuronal activity occurs. Activation of the NMDA channel plays a major role in cognition, chronic pain, opioid tolerance, and mood regulation, and it is considered the principal receptor involved in the phenomena of central sensitisation and windup (26-30). Mackey reported in 2007 that, in animal studies of neuropathic pain, an increase in the expression of NMDA receptors was demonstrated to play a role in clinical chronic neuropathic pain, including CRPS (6). Therefore, it is reasonable to hypothesise that NMDA receptor antagonism would be beneficial for CRPS. Agents that have clinically relevant NMDA-blocking properties include ketamine, amantadine, memantine, dextromethorphan, and methadone (6).

Amantadine hydrochloride is chemically designated as 1-adamantanamine hydrochloride. Amantadine hydrochloride has pharmacological actions as both an anti-Parkinson and an antiviral drug. The action mechanisms of Amantadine in the treatment of Parkinson's disease and drug-induced extrapyramidal reactions are yet unknown. Amantadine may have direct and indirect effects on dopamine neurons. Further, amantadine is a weak, non-competitive NMDA receptor antagonist. 


\section{AN ARGUMENT FOR THE USE OF AMANTADINE TO TREAT COMPLEX REGIONAL PAIN SYNDROME}

Ossola presented data from both animal and human evidence, demonstrating that amantadine may exert neuroprotection in several neurological disorders (31). It is generally believed that this neuroprotection results from the ability of amantadine to inhibit glutamatergic NMDA receptor (31). Further, amantadine protected rat midbrain cultures from $\mathrm{MPP}^{+}$(1-methyl-4-phenylpyridinium), a positively charged organic molecule and neurotoxin that acts by interfering with oxidative phosphorylation in mitochondria by inhibiting complex I, leading to the depletion of Adenosine triphosphate (ATP) and eventual cell death, and lipopolysaccharide (LPS); $\mathrm{MPP}^{+}$ and LPS are two toxins commonly used in Parkinson's disease models. Their studies revealed that amantadine reduced LPS- and $\mathrm{MPP}^{+}$-induced toxicity of the dopamine neuron through the inhibition of the release of microglial proinflammatory factors as well as increased the expression of neurotrophic factors such as Glial cell-derived neurotrophic factor (GDNF) from astroglia (31). Last, different from the general view on amantadine's action, they provided evidence suggesting that NMDA receptor inhibition is not crucial for the neuroprotective effect of amantadine (31). They concluded that amantadine protected the dopamine neurons in two Parkinson's disease models through a novel dual mechanism, reducing the release of proinflammatory factors from activated microglia and increasing the expression of GNDF in astroglia (31).

Moore provided data to validate amantadine's ability to decrease central sensitisation and opioid tolerance in some patients and enhance the effects of NSAIDS, gabapentin, and opioids in dog models (32-34). However, a randomised, placebo-controlled trial evaluated the use of amantadine in conjunction with meloxicam in dogs with osteoarthritis and showed significant improvement in pain scores for dogs receiving amantadine as compared to placebo (33). Amantadine is an NMDA antagonist that has shown mixed results in the treatment of neuropathic pain in humans (34).

Kleinböhl investigated whether the established psychophysical measures of enhanced experimental sensitisation in chronic musculoskeletal pain can be reduced by adjuvant treatment with an NMDA receptor antagonist, amantadine sulfate, and whether a reduction in sensitisation might be accompanied by a concurrent improvement in clinical pain (35) . He examined 26 patients with chronic back pain who were included in the randomised, double-blind, placebocontrolled study, and they received daily dosages of either placebo or $100 \mathrm{mg}$ of amantadine sulphate during a 1-week treatment. The participants completed quantitative sensory testing of pain thresholds and experimental sensitisation before and after treatment and obtained their clinical pain ratings before, during, and after treatment (35). It was concluded that adjuvant chronic pain treatment with NMDA receptor antagonists might be beneficial for chronic pain if enhanced sensitisation is involved (35).

Meythaler et al. examined traumatic brain injury (TBI) caused by a high-speed transportation accident that had a mechanism of injury described as diffuse axonal injury, which is associated with a reduction in dopamine turnover in the brain (36). Due to its effect on both dopamine and NMDA channels, amantadine has been the subject of considerable interest in clinical use in acute TBI (36). Meythaler et al. studied amantadine (200 mg) and placebo which were each administered for 6 weeks (12 weeks in total) to consecutively recruited patients. In this study, 35 subjects with TBI due to transportation accidents and a Glasgow coma scale score of 10 or less within the first 24 hours after admission were randomly assigned to a double-blind, placebocontrolled, crossover design trial (36). There was a consistent trend toward a more rapid functional improvement regardless of when a patient with diffuse axonal injury (DAI)-associated TBI was started on amantadine in the first 3 months after injury (36).

Fujita et al. relate an interesting case report describing a 59-year-old Japanese woman who suffered from necrotising fasciitis in her right lower limb and underwent amputation of the limb at the hip joint (37). The patient experienced phantom sensations in the right lower limb, dysesthesia, and stump pain immediately after surgery (37). They began pregabalin treatment (oral $150 \mathrm{mg} /$ day) on postoperative day 3 , but the phantom limb sensation and dysesthesia did not subside (38). Then, the clinicians began oral amantadine at a dose of $200 \mathrm{mg} /$ day on postoperative day 8 , and her symptoms gradually improved (37). Mexiletine (oral $200 \mathrm{mg} /$ day) was 
started on postoperative day 31 , and symptoms further alleviated. In the combined therapy with pregabalin (oral $450 \mathrm{mg} /$ day), amantadine (oral 450 $\mathrm{mg} /$ day), and mexiletine (oral $300 \mathrm{mg}$ /day) to treat phantom limb pain, amantadine was particularly effective (37). These investigators declared that the positive outcome for the patient suggests that amantadine is an effective treatment option for phantom limb pain and phantom limb sensation after limb amputation (37).

Nicol et al. advocated for the use of NMDA antagonists as one of the treatments for CRPS, as they offer alternative pharmacological agents to replace opioids (38). Intravenous ketamine results in significantly better pain relief when compared to placebo (39). In this study, ketamine was administered over a 4-day period. The dose was given in an individualised, stepwise fashion starting at 1.2 mcg $\mathrm{kg}^{-1} \mathrm{~min}$ (approximately $5 \mathrm{mg} / \mathrm{h}$ for a $70 \mathrm{~kg}$ patient) to a maximum of $7.2 \mathrm{mcg} \mathrm{kg}^{-1} \min (30$ $\mathrm{mg} / \mathrm{h}$ for a $70 \mathrm{~kg}$ patient). Ketamine was noted to be significantly better in terms of pain relief. However, the difference was gone at 12 weeks, and there was no difference between the treatment groups in their secondary outcomes. Another study showed the superiority of ketamine infusion over placebo in relieving pain, reducing allodynia and thermal and deep pressure pain thresholds, and improving motor function (40). Further, Nicol et al. have described the use of memantine as a prospective open series, evidencing pain reduction among patients with CRPS but with the limitation that the investigators used the van de Beek criteria to diagnose CRPS $(39,41,42)$. This led the investigators to compare morphine (30 $\mathrm{mg}$ daily) with or without memantine (40 mg daily) in a prospective, randomised, controlled, and blinded design (42). The investigators showed that only a combination of morphine and memantine reduced the pain and disability (42). Finally, Birklein and Dimova declared that pharmacological treatments may be particularly effective in acute stages and include steroids, bisphosphonates, dimethyl sulfoxide cream, anti-neuropathic pain drugs and intravenous longterm ketamine administration. These have shown efficacy in randomised controlled trials (43). However, repeated ketamine application is demanding and has side effects (43) [43]. Important components of the treatment include physio- and occupational therapies including behavioural therapy (43).

\section{6 | COMPARISONS OF NMDA RECEPTOR ANTAGONISTS}

Birklein and Dimova proclaimed that there is no form of efficacy in CRPS for drugs used in other chronic neuropathic pain disorders (43). Memantine was first synthesised by Eli Lilly and Company and patented in 1968 as a derivative of amantadine (44). The efficacy of memantine in neurodegenerative diseases and its ability to protect neurons in animal models of both acute and chronic neurologic disorders suggest that memantine and drugs acting in a similar manner could become important new weapons in the fight against neuronal damage (43, 44). Building on this assertion offered by Lipton, an inference that amantadine can be used to fight against neuronal damage can be offered based on examining both the medications' chemical structures. (44) Wertli et al. concluded, after analysing rational pain management of CRPS-I using a network meta-analysis, that while most medications showed some efficacy on short-term follow-up, only bisphosphonates, NMDA analogs (which include both memantine and ketamine), and vasodilators showed better long-term pain reduction than placebo (45). Figure 2 presents the chemical structures of amantadine, memantine, and ketamine for an appreciation of their stereo-chemical similarities. Based on a logical argument grounded in the previously presented data related to the efficiency of amantadine, memantine, and ketamine in treating pain syndromes, including CRPS, as well as their stereo-chemical structural similarities, it can be declared that amantadine may offer an advantage when treating CRPS. It is recommended that further efficient prospective, randomised, controlled, and blinded design investigations centred on the use of amantadine to treat CRPS should be conducted. 


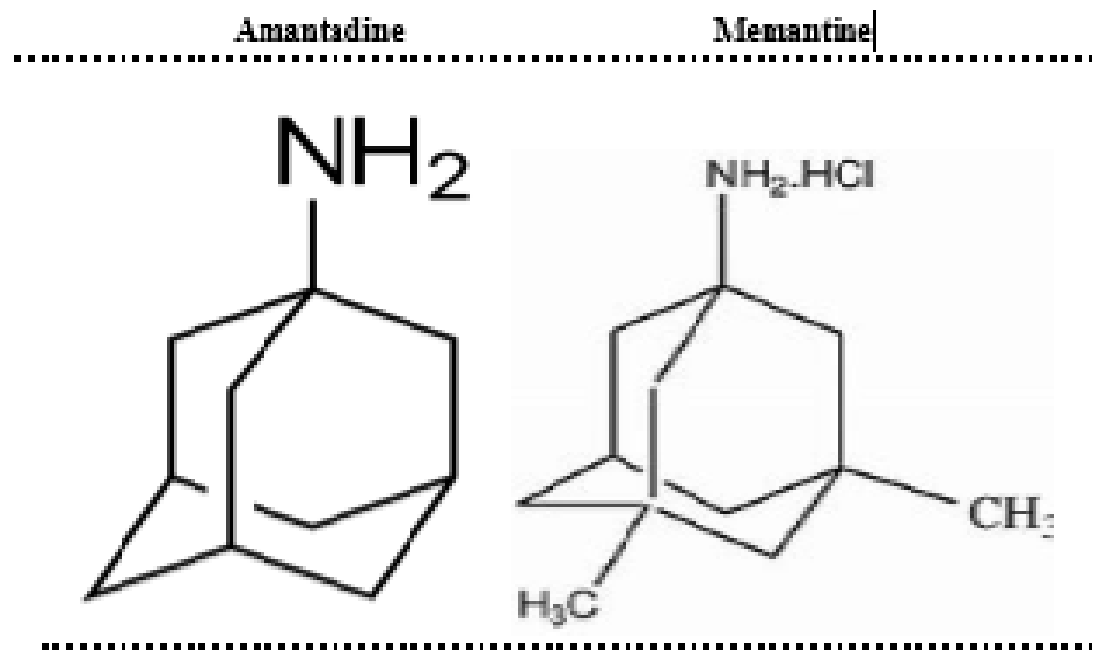

Ketamine

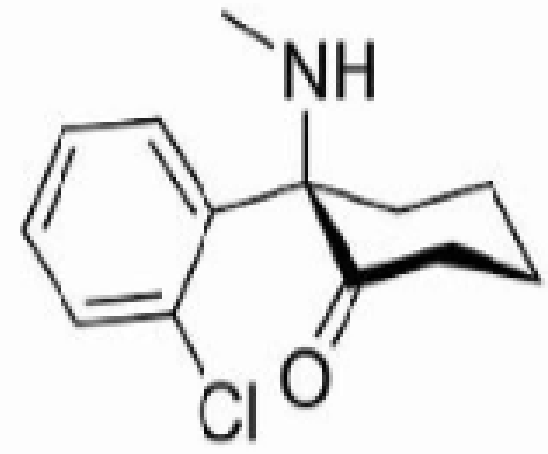

FIGURE 2: Chemical Structures of Amantadine, Memantine andKetamine

\section{7 | CONCLUSIONS}

The results of a literature search focused on amantadine, memantine, and ketamine as treatments for complex regional pain syndrome were described in this paper. In addition, the pharmacology of amantadine as it applies to the inflammation and pathology of CRPS was presented. Further, literature accounts centred on the effective use of amantadine to treat pain syndromes were described. Finally, comparisons of amantadine, memantine, and other NMDA receptor antagonists used to treat CRPS were presented.

\section{REFERENCES}

1. Eldufani J, Elahmer N, Blaise G. A medical mystery of complex regional pain syndrome. Heliyon. 2020; 6(2):e03329.

2. Marinus J, Moseley GL, Birklein F, et al. Clinical features and pathophysiology of complex regional pain syndrome. Lancet Neurol. 2011; 10(7):637-648.

3. Bruehl S, Harden RN, Galer BS, et al. External validation of IASP diagnostic criteria for Complex Regional Pain Syndrome and proposed research diagnostic criteria. International Association for the Study of Pain. Pain. 1999; 81(1-2):147-154. 
4. Complex Regional Pain Syndrome Fact Sheet. National Institute of Neurological Disorders and Stroke. Department of Health and Human Services, Bethesda, Maryland, 2013, 20892-2540. https://www.ninds.nih.gov/ Disorders/Patient-Caregiver-Education/FactSheets/Complex-Regional-Pain-SyndromeFact-Sheet. Accessed July 13, 2020.

5. Acerra NE, Souvlis T, Moseley GL. Stroke, complex regional pain syndrome and phantom limb pain: can commonalities direct future management? J Rehabil Med. 2007; 39(2):109114.

6. Mackey S, Feinberg S. Pharmacologic therapies for complex regional pain syndrome. Curr Pain Headache Rep. 2007 Feb; 11(1):3843.

7. Mitchell SW, Morehouse GR, Keen WW. Gunshot wounds and other injuries of nerves. Philadelphia: JB Lippincott \& Co., 1864.

8.Sudeck P. Über die akute (reflektorische) Knochenatrophie nach Entzündungen und Verletzungen in den Extremitäten und ihre klinischen Erscheinungen. Fortschr Röntgenstr. 1901; 5:227-293.

9. Evans JA. Reflex sympathetic dystrophy. Surg Clin North Am. 1946; 26:780-790.

10. Stanton-Hicks M, Jänig W, Hassenbusch S, et al. Reflex sympathetic dystrophy: Changing concepts and taxonomy. Pain. 1995; 63:127133.

11.Kozin F, Ryan LM, Carerra GF, et al. The reflex sympathetic dystrophy syndrome (RSDS). III. Scintigraphic studies, further evidence for the therapeutic efficacy of systemic corticosteroids, and proposed diagnostic criteria. Am J Med. 1981; 70:23-30.
12. Veldman PH, Reynen HM, Arntz IE, et al. Signs and symptoms of reflex sympathetic dystrophy: Prospective study of 829 patients. Lancet. 1993; 342:1012-1016.

13. van de Beek WJ, Schwartzman RJ, van Nes SI, et al. Diagnostic criteria used in studies of reflex sympathetic dystrophy. Neurology. 2002; 58:522-526.

14. Bruehl S, Harden RN, Galer BS, et al. External validation of IASP diagnostic criteria for Complex Regional Pain Syndrome and proposed research diagnostic criteria. International Association for the Study of Pain. Pain. 1999; 81:147-154.

15. Harden RN, Bruehl S, Perez RS, et al. Validation of proposed diagnostic criteria (the "Budapest Criteria") for complex regional pain syndrome. Pain. 2010; 150:268-74.

16. Benzon HT, Liu SS, Buvanendran A. Evolving definitions and pharmacologic management of complex regional pain syndrome. Anesth Analg. 2016; 122:601-604.

17. Schmelz M, Michael K, Weidner C, et al. Which nerve fibers mediate the axon reflex flare in human skin? NeuroReport. 2002; 11(3):645-648.

18. Borchers AT, Gershwin ME. Complex regional pain syndrome: A comprehensive and critical review. Autoimmun Rev. 2014; 13(3):242-265.

19. Calder JS, Holten I, McAllister RM. Evidence for immune system involvement in reflex sympathetic dystrophy. J Hand Surg. 1998; (2):147-150. 
20. Braus DF, Krauss JK, Strobel J. The shoulder-hand syndrome after stroke: A prospective clinical trial. Ann Neurol: Off J Am Neurol Assoc Child Neurol Soc. 1994; 36(5):728-733.

21. Cheng JK, Ji RR. Intracellular signaling in primary sensory neurons and persistent pain. Neurochem Res. 2008; 33(10):1970-1978.

22. Birklein F, Schmelz M. Neuropeptides, neurogenic inflammation, and complex regional pain syndrome (CRPS). Neurosci Lett. 2008; 437(3):199-202.

23. Herbert MK, Holzer P. Neurogenic inflammation. I. Basic mechanisms, physiology, and pharmacology. Anasthesiol Intensivmed Notfallmed Schmerztherapie: AINS. 2002; 37(6):314-325.

24. König S, Schlereth T, Birklein F. Molecular signature of complex regional pain syndrome (CRPS) and its analysis. Expert Rev Proteomics. 2017; 14(10):857-867.

25. Kingery WS. Role of neuropeptide, cytokine, and growth factor signaling in complex regional pain syndrome. Pain Med. 2010; 11(8):1239-1250.

26. Cohen SP, Liao W, Gupta A, et al. Ketamine in pain management. Adv Psychosom Med. 2011; 30:139-161.

27. Chang $\mathrm{CH}$, Hsiao $\mathrm{YH}$, Chen $\mathrm{YW}$, et al. Social isolation-induced increase in NMDA receptors in the hippocampus exacerbates emotional dysregulation in mice. Hippocampus. $2015 ; 25: 474-485$.

28. Duman RS, Li N, Liu RJ, et al. Signaling pathways underlying the rapid antidepressant actions of ketamine. Neuropharmacology. 2012; $62: 35-41$.
29. Trujillo KA, Akil H. Inhibition of opiate tolerance by non-competitive N-methyl-daspartate receptor antagonists. Brain Res. 1994; 633:178-188.

30. Zhou HY, Chen SR, Pan HL. Targeting Nmethyl-d-aspartate receptors for treatment of neuropathic pain. Expert Rev Clin Pharmacol. 2011; 4:379-388.

31. Ossola B, Schendzielorz N, Chen SH, et al. Amantadine protects dopamine neurons by a dual action: reducing activation of microglia and inducing expression of GDNF in astroglia [corrected] [published correction appears in Neuropharmacology. 2012 Feb; 62(2):1162]. Neuropharmacology. 2011; 61(4):574-582.

32. Moore SA. Managing Neuropathic Pain in Dogs. Front Vet Sci. 2016 Feb 22; 3:12.

33. Collins S, Sigtermans MJ, Dahan A, et al. NMDA receptor antagonists for the treatment of neuropathic pain. Pain Med. 2010; 11:1726-42.

34. Lascelles BD, Gaynor JS, Smith ES, et al. Amantadine in a multimodal analgesic regimen for alleviation of refractory osteoarthritis pain in dogs. J Vet Intern Med. 2008; 22:53-59.

35. Kleinböhl D, Görtelmeyer R, Bender HJ, et al. Amantadine sulfate reduces experimental sensitization and pain in chronic back pain patients. Anesth Analg. 2006; 102(3):840-847.

36. Meythaler JM, Brunner RC, Johnson A, et al. Amantadine to improve neurorecovery in traumatic brain injury-associated diffuse axonal injury: a pilot double-blind randomized trial. J Head Trauma Rehabil. 2002; 17(4):300-313.

37. Fujita M, Inomata S, Takao I, et al. Pharmacotherapy with amantadine and mexiletine was effective for phantom limb pain that developed after lower-limb amputation surgery. Jap Soc of Pain Clinicians. 2020; 27(2):163-166. 
38. Nicol AL, Hurley RW, Benzon HT. Alternatives to opioids in the pharmacologic management of chronic pain syndromes: A narrative review of randomized, controlled, and blinded clinical trials. Anesth Analg. 2017; 125(5):1682-1703.

39. Sigtermans MJ, van Hilten JJ, Bauer MC, et al. Ketamine produces effective and long-term pain relief in patients with complex regional pain syndrome type 1. Pain. 2009; 145:304-11.

40. Schwartzman RJ, Alexander GM, Grothusen JR, et al. Outpatient intravenous ketamine for the treatment of complex regional pain syndrome: a double-blind placebocontrolled study. Pain. 2009; 147:107-15.

41. Sinis N, Birbaumer N, Gustin S, et al. Memantine treatment of complex regional pain syndrome: a preliminary report of six cases. Clin J Pain. 2007; 23:237-43.
42. Gustin SM, Schwarz A, Birbaumer N, et al. NMDA-receptor antagonist, and morphine decrease CRPS-pain and cerebral pain representation. Pain. 2010; 151:69-76.

43. Birklein F, Dimova V. Complex regional pain syndrome-up to date. Pain Rep. 2017; 2(6):e624.

44. Lipton SA. Failures and successes of NMDA receptor antagonists: Molecular basis for the use of open-channel blockers like memantine in the treatment of acute and chronic neurologic insults. NeuroRx. 2004; 1(1):101110.

45. Wertli MM, Kessels AG, Perez RS, et al. Rational pain management in complex regional pain syndrome 1 (CRPS 1) - A network metaanalysis. Pain Med. 2014; 15(9):1575-1589.

How to cite this article: Smith R.G. An Argument for the Use of Amantadine to Treat Complex Regional Pain Syndrome. Journal of Current Medical Research and Opinion. 2020;692-701. https://doi. org/10.15520/jcmro.v3i10.353 